\title{
MicroRNA-7-5p regulates the proliferation and migration of intestinal epithelial cells by targeting trefoil factor 3 via inhibiting the phosphoinositide 3-kinase/Akt signalling pathway
}

\author{
JING GUO, LINGFEN XU, XU TENG and MEI SUN
}

Department of Pediatrics, The Affiliated Shengjing Hospital, China Medical University, Shenyang, Liaoning 110004, P.R. China

Received April 12,2017; Accepted August 21, 2017

DOI: $10.3892 /$ ijmm.2017.3120

\begin{abstract}
Trefoil factor 3 (TFF3) reconstructs the epithelial barrier by stimulating epithelial cell migration and proliferation, and significantly contributes to intestinal mucosal damage and healing. In a previous study, TFF3 was identified as a novel target of microRNA-7-5p (miR-7-5p). The aim of the present study was to investigate the roles and mechanisms of miR-7-5p in the proliferation and migration of intestinal epithelial cells. Reverse transcription-quantitative polymerase chain reaction (RT-qPCR) was performed to determine the expression level of miR-7-5p in the experimental groups. In addition, western blot analysis was performed to examine the expression levels of TFF3, phosphoinositide 3-kinase (PI3K), Akt and phosphorylated (p)-AKT when miR-7-5p or TFF3 was overexpressed, and the effects of miR-7-5p and TFF3 on LS174T cell proliferation and migration were simultaneously investigated. miR-7-5p was demonstrated to decrease the expression level of TFF3, and inhibit LS174T cell proliferation and migration, which was accompanied by decreased expression levels of PI3K and p-Akt. miR-7-5p was decreased following combined treatment with the TFF3 plasmid and miR-7-5p mimics, compared with treatment with miR-7-5p mimics alone, which was accompanied by increased expression levels of TFF3, PI3K and p-Akt, and enhanced LS174T cell proliferation and migration effects. The expression levels of miR-7-5p in the miRNA negative control (NC) + LY294002 group, the miR-7-5p mimic + LY294002 group, and the miR-7-5p mimic + TFF3 plasmid + LY294002 group were higher than those in the NC group, the miR-7-5p mimic group and the miR-7-5p mimic + TFF3 plasmid group, respectively. Accordingly, the expression level of TFF3 was downregulated and the proliferation and migration ability of the cells was downregulated. The present study demonstrates that overexpressed miR-7-5p may inhibit the proliferation and
\end{abstract}

Correspondence to: Professor Mei Sun, Department of Pediatrics, The Affiliated Shengjing Hospital, China Medical University, No. 36 Sanhao Street, Shenyang, Liaoning 110004, P.R. China E-mail: sunm@sj-hospital.org

Key words: microRNA-7-5p, trefoil factor 3, proliferation, migration, LS174T migration of LS174T cells by targeting the expression of TFF3 via inhibiting the PI3K/Akt signalling pathway. The PI3K/Akt signalling pathway may exert a feedback regulation effect on miR-7-5p, inhibiting the activity of this signalling pathway, which increases the miR-7-5p expression levels and further enhances the effects of miR-7-5p on cell proliferation and migration.

\section{Introduction}

Inflammatory bowel disease (IBD), including Crohn's disease and ulcerative colitis, is characterized by chronic intestinal inflammation resulting from a complex interaction of genetically susceptible hosts, disruption of mucosal barriers, an abnormal immune response to environmental factors, and disturbance of the intestinal flora (1). The pathogenesis of IBD remains unclear, although studies in IBD animal models and IBD patients have identified a common pathologic outcome: structural and functional impairment of the intestinal mucosal barrier (2-4). The changes in intestinal epithelial mucosal barrier function are important in the occurrence, development and prognosis of IBD. Dysfunction of intestinal epithelial tight junctions leads to immune activation and promotes the occurrence of colitis (5). Clinically, the degree of mucosal repair has become the standard for evaluating the therapeutic effect of IBD (2).

The key phenomenon in the early repair of epithelial cells following injury is cell migration, whereby normal epithelial cells surrounding the damaged area migrate to the injured area, and subsequently reconstruct and maintain epithelial integrity, which is referred to as the rapid repair pathway (6). Slow repair predominantly refers to the process of cell proliferation. Cell proliferation primarily repairs the damaged mucosa by increasing the number of mitotic cells (7). Trefoil factor 3 (TFF3) is a small molecule polypeptide that is secreted by intestinal goblet cells and significantly contributes to the protection of intestinal mucosal integrity, the reconstruction and repair of intestinal mucosal injury and the anti-apoptosis of intestinal mucous cells $(8,9)$.

Numerous studies have indicated that TFF3 promotes the migration of various types of cells in vitro and in vivo, and that the migration mechanism may be closely associated with the APC protein, $\beta$-catenin, E-cadherin, the epidermal growth factor (EGF) receptor complex and extracellular 
signal-regulated kinase (ERK). It has been found that repair of the epithelium by TFF3 is independent of transforming growth factor- $\beta$ and that the epithelium is precisely repaired by promoting the migration of cells surrounding the injured area to the damaged site (10). In addition, TFF3 promotes the migration of epithelial cells by affecting the expression and localization of catenin in epithelial cells, and by inducing phosphorylation of catenin, leading to a decrease in tight junctions between cells $(11,12)$. The EGF and Ras signalling pathways may also be involved in the mechanism through which TFF3 promotes intestinal epithelial cell migration. However, the upstream regulatory mechanism of TFF3 remains unclear.

In a previous study, TFF3 was demonstrated to be a novel target of miR-7-5p (13). miR-7 has been investigated primarily in tumour diseases. Numerous reports have indicated that miR-7 affects the functions of cell metabolism, growth, proliferation and apoptosis through acting on different target proteins $(14,15)$. Therefore, we were interested in further examining the effects of miR-7-5p on TFF3 and the relationship among miR-7-5p, TFF3 and the proliferation and migration of intestinal epithelial cells. In the present study, we hope to provide a theoretical basis for the mechanism and treatment of IBD.

\section{Materials and methods}

Cell culture experiments. The human colonic epithelial cell line LS174T was obtained from the American Type Culture Collection (ATCC; Manassas, VA, USA). The cell line was grown to near confluence in Dulbecco's modified Eagle's medium supplemented with $10 \%$ fetal bovine serum (FBS; all from HyClone Laboratories, GE Healthcare Life Sciences, Logan, UT, USA), $50 \mathrm{U} / \mathrm{ml}$ penicillin and $50 \mathrm{mg} / \mathrm{ml}$ streptomycin at $37^{\circ} \mathrm{C}$. The cells were subcultured following partial digestion with $0.25 \%$ trypsin and $0.9 \mathrm{mmol} / 1$ EDTA in $\mathrm{Ca}^{2+}$ free and $\mathrm{Mg}^{2+}$-free phosphate-buffered saline (PBS).

TaqMan assays. To evaluate the expression level of miR-7-5p in the LS174T cells, total RNA was isolated from LS174T cells using the mirVana miRNA isolation kit (Thermo Fisher Scientific, Inc., Waltham, MA, USA). Reverse transcription (RT) was performed using the TaqMan miRNA reverse transcription kit (Thermo Fisher Scientific, Inc.) with a stem loop-specific RT primer, followed by TaqMan PCR analysis with small RNA-specific primers for miR-7-5p and U6 small nucleolar RNA (snRNA; Thermo Fisher Scientific, Inc.). Briefly, RT was performed as follows: the RT Master Mix included $100 \mathrm{nM}$ dNTPs with $0.15 \mu \mathrm{l}$ dTTP, MutiScribeTM Reverse Transcription $(50 \mathrm{U} / \mu 1 ; 1 \mu \mathrm{l}), 10 \mathrm{X}$ reverse buffer (1.5 $\mu \mathrm{l}), 0.19 \mu \mathrm{l}$ RNase inhibitor $(20 \mathrm{U} / \mu \mathrm{l})$ and $4.16 \mu \mathrm{l}$ nucleasefree water. RT Master Mix ( $7 \mu \mathrm{l})$ was combined with $5 \mu \mathrm{l}$ total RNA (1-10 ng), and $3 \mu \mathrm{l}$ RT primer (5X) was added, mixed and centrifuged at $1000 \mathrm{x} \mathrm{g}$ at $4^{\circ} \mathrm{C}$ for $5 \mathrm{sec}$. The following parameter values were used to program the thermal cycler: $16^{\circ} \mathrm{C}$ temperature bath for $30 \mathrm{~min}, 42^{\circ} \mathrm{C} 30 \mathrm{~min}$ for RT reaction, $85^{\circ} \mathrm{C}$ heating for $5 \mathrm{~min}$ to terminate the reaction, and setting the temperature at $4^{\circ} \mathrm{C}$ for running qPCR. According to the manufacturer's instructions, qPCR was performed to measure the expression levels of miR-7-5p and U6 using the Universal PCR Master Mix kit (Thermo Fisher Scientific, Inc.) and specific TaqMan probes (Thermo Fisher Scientific, Inc.). The
PCR protocol was as follows: $20 \mu 1$ reaction volume, including $10 \mu 1$ TaqMan Universal PCR Master Mix II no $\mathrm{UNG}^{+}$(Thermo Fisher Scientific, Inc.; cat. no. 4440049), $7.67 \mu 1$ nuclease-free water, $1.0 \mu \mathrm{l}$ TaqMan Small RNA assay (20X) and $1.33 \mu 1$ product from the RT reaction. The thermocycling conditions were as follows: optional AmpErase UNG activity, $50^{\circ} \mathrm{C}$ for $2 \mathrm{~min}$; enzyme activation, $95^{\circ} \mathrm{C}$ for $10 \mathrm{~min}$ and PCR was performed for 40 cycles. Denaturing was performed at $95^{\circ} \mathrm{C}$ for $15 \mathrm{sec}$ and extension was performed at $60^{\circ} \mathrm{C}$ for $60 \mathrm{sec}$. The relative expression levels of $\mathrm{miR}-7-5 \mathrm{p}$ were normalized to the expression of U6 RNA via the $2^{-\Delta \Delta \mathrm{Ct}}$ method (16). The measurements were performed in triplicate.

Plasmid construction and transfection. The miR-7-5p mimic, miRNA negative control (NC), miR-7-5p inhibitor and miR-7-5p inhibitor NC were purchased from Guangzhou RiboBio Co., Ltd. (Guangzhou, China). The sequences were as follows: 5'-UGGAAGACUAGUGAUUUUGUUGU-3' for miR-7-5p mimic; 5'-UUUGUACUACACAAAAGUACUG-3' for miR-7-5p mimic NC; 5'mAmCmAmAmCmAmAmAm AmUmCmAmCmUmAmGmUmCmUmUmCmCmA3' for miR-7-5p inhibitor; and 5'-mCmAmGmUmAmCmUmUm UmUmGmUmGmUmAmGmUmAmCmAmAmA-3' for miR-7-5p inhibitor NC. The LS174T cells were seeded at a density of $4 \times 10^{5}$ cells/well in 6 -well plates at $37^{\circ} \mathrm{C}$ and transfected with $100 \mathrm{nM}$ miR-7-5p mimic, miRNA NC, miR-7-5p inhibitor NC, and miR-7-5p inhibitor of each at a ratio of 1:2 and $2 \mu \mathrm{g}$ TFF3 $(545.36 \mu \mathrm{g} / \mathrm{ml})$. The TFF3 plasmid was purchased from SinoBiological, Inc. (Beijing, China). Transfection was performed using Lipofectamine 3000 (Thermo Fisher Scientific, Inc.) according to the manufacturer's instructions. Cells were harvested after $48 \mathrm{~h}$ and subjected to various assays.

Western blot analysis. Proteins from the transfected cells were harvested at $48 \mathrm{~h}$ post-transfection using radioimmune precipitation assay buffer $(50 \mathrm{mM}$ Tris, $\mathrm{pH} 7.4,150 \mathrm{mM}$ $\mathrm{NaCl}, 1 \%$ Triton $\mathrm{X}-100,1 \%$ sodium deoxycholate and $0.1 \%$ SDS and inhibitors, including sodium orthovanadate, sodium fluoride, EDTA and leupeptin, as well as proteinase/ phosphatase inhibitors) for $30 \mathrm{~min}$ on ice. Equal quantities of protein $(10-20 \mu \mathrm{g})$ were separated on $8-15 \%$ SDS-PAGE gels and transferred to polyvinylidene fluoride membranes (EMD Millipore, Billerica, MA, USA) at $80 \mathrm{v}, 1.5 \mathrm{~h}$. The protein expression levels of phosphoinositide 3-kinase (PI3K), Akt, phosphorylated (p)-Akt, TFF3 and glyceraldehydes3-phosphate dehydrogenase (GAPDH) were detected using specific antibodies of anti-PI3K (rabbit polyclonal antibody; dilution, 1:1,000; cat. no. WL01169; Wanleibio, Shenyang, China), anti-AKT (rabbit polyclonal antibody; dilution, 1:1,000; cat. no. WL0003b; Wanleibio), anti-p-AKT (rabbit polyclonal antibody; dilution, 1:1,000; cat. no. WLP001; Wanleibio), anti-TFF3 (rabbit monoclonal antibody; dilution, 1:1,000; cat. no. ab108599; Abcam, Cambridge, MA, USA) and anti-GAPDH (rabbit monoclonal antibody; dilution, 1:5,000; cat. no. ab9485; Abcam). After washing in Tris-buffered saline with Tween-20 (0.1\%) (Sigma-Aldrich, St. Louis, MO, USA), membranes were incubated with the secondary antibody [horseradish peroxidase-labeled goat anti-rabbit immunoglobulin $\mathrm{G}(\mathrm{H}+\mathrm{L})$; dilution, 1:2,000; cat. no. A0208; 
Beyotime Institute of Biotechnology, Haimen, China] at room temperature for $2 \mathrm{~h}$. Bands were visualized using the enhanced chemiluminescence method (Pierce ${ }^{\mathrm{TM}}$ ECL Western Blotting Substrate; Thermo Fisher Scientific, Inc.) according to the manufacturer's protocol. Semi-quantification was performed using the ImageJ v1.48u software (National Institutes of Health, Bethesda, Maryland, USA).

Cell proliferation assay. The 3-(4,5-dimethylthiazol-2-yl)2,5-diphenyltetrazolium bromide (MTT) assay was used to measure cell proliferation. At $0,24,48$ and $72 \mathrm{~h}$ post-transfection, the transfection medium was replaced with $150 \mu \mathrm{l}$ of fresh serum-free medium containing $0.5 \mathrm{~g} / 1 \mathrm{MTT}$ in each well. Following incubation at $37^{\circ} \mathrm{C}$ for $4 \mathrm{~h}$, the MTT medium was removed via aspiration, and $50 \mu 1$ dimethyl sulfoxide (DMSO) was added to each well. Subsequent to incubation at $37^{\circ} \mathrm{C}$ for a further $10 \mathrm{~min}$, the absorbance $(490 \mathrm{~nm})$ of each sample was measured using a plate reader (BioTek Instruments, Inc., Winooski, VT, USA). This experiment was repeated 3 times.

Cell migration assay. The migration ability of LS174T cells was evaluated using 24-well Transwell chambers (EMD Millipore, Boston, MA, USA). For all groups, LS174T cells were diluted in serum-free medium at the logarithmic growth phase. The concentration of the cell suspension was adjusted to $5 \times 10^{4}$ cells $/ \mathrm{ml}$, and $200 \mu 1$ of the suspension was then inoculated into the upper Transwell chamber. The cells were subsequently incubated for $24 \mathrm{~h}$ at $37^{\circ} \mathrm{C}$ and $5 \% \mathrm{CO}_{2}$ incubator, fixed with $4 \%$ paraformaldehyde at room temperature for $20 \mathrm{~min}$ and stained with $0.5 \%$ crystal violet dye for $5 \mathrm{~min}$ at room temperature. After washing various times with water, the number of cells that had migrated through the filter into the lower wells was counted using an inverted microscope, and 5 fields were selected and calculated the arithmetic mean value.

Experimental grouping. To illustrate the interaction of miR-7-5p, TFF3 and the PI3K/Akt signalling pathway in the regulation of $\mathrm{LS} 174 \mathrm{~T}$ cell proliferation and migration by miR-7-5p, a PI3K/Akt signalling pathway inhibitor (LY294002) and a TFF3-overexpressing plasmid were used. The LS174T cells were divided into six groups as follows: i) miRNA NC group, when the LS174T cells were $60-80 \%$ confluent, they were transfected with the miRNA mimic NC for $48 \mathrm{~h}$ and harvested; ii) miR-7-5p mimic experimental group, when the LS174T cells were $60-80 \%$ confluent, the cells were harvested after transfection with miR-7-5p mimics for $48 \mathrm{~h}$; iii) miR-7-5p mimic + TFF3 overexpression vector transfection group, when the LS174T cells were $60-80 \%$ confluent, the cells were transfected with the TFF3 plasmid together with miR-7-5p mimics and were harvested following co-culture for $48 \mathrm{~h}$; iv) miRNA mimic NC + LY294002 treatment group, $24 \mathrm{~h}$ after the cells were transfected with the miRNA mimic NC, they were treated with the PI3K inhibitor, LY294002 (50 $\mu \mathrm{M})$ for $24 \mathrm{~h}$; v) miR-7-5p mimic + LY294002 treatment group, $24 \mathrm{~h}$ after the cells were transfected with miR-7-5p mimics, they were treated with $50 \mu \mathrm{M}$ LY294002 for $24 \mathrm{~h}$; and vi) miR-7-5p mimics + TFF3 overexpression plasmid + LY294002 treatment group, the cells were transfected with miR-7-5p mimics and TFF3 plasmids for $24 \mathrm{~h}$ and treated with $50 \mu \mathrm{M}$ LY294002 for $24 \mathrm{~h}$.

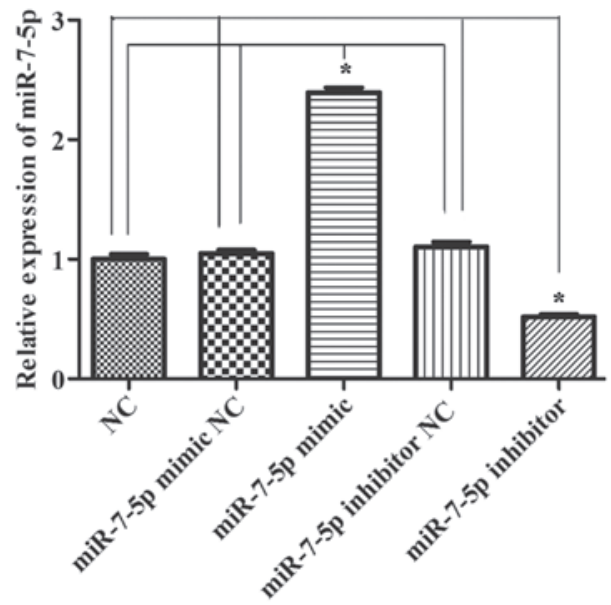

Figure 1. Expression levels of miR-7-5p following transfection with miR-7-5p mimics and miR-7-5p inhibitor were detected by reverse transcriptionquantitative polymerase chain reaction. U6 served as a control. All values are presented as the means \pm standard deviation of three replicates. ${ }^{*} \mathrm{P}<0.05$. miR, microRNA; NC, negative control.

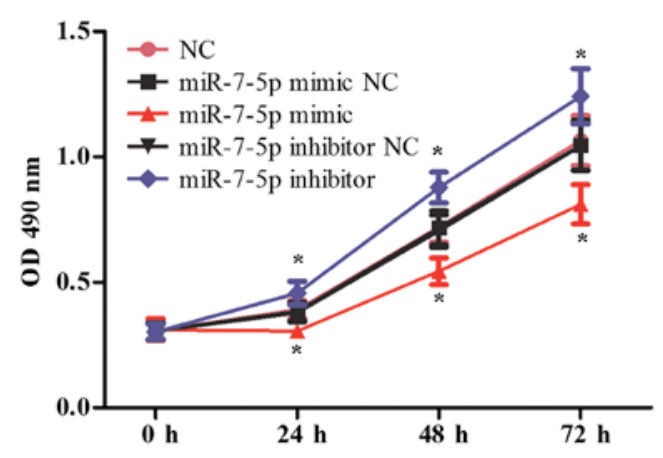

Figure 2. Effects of miR-7-5p on proliferation of LS174T cells. 3-(4,5-dimethylthiazol-2-yl)-2,5-diphenyltetrazolium bromide assay was performed to determine the effect of overexpression and downregulation of miR-7-5p on cell proliferation of LS174T cells. The assays were performed in triplicate. All values are presented as the means \pm standard deviation of three replicates. ${ }^{*} \mathrm{P}<0.05$ compared with NC, miR-7-5p mimic NC and miR-7-5p inhibitor NC groups. miR, microRNA; NC, negative control; OD, optical density.

Statistical analysis. All values were expressed as means \pm standard error of the mean. Differences between the groups were analysed via the one-way ANOVA, followed by the Bonferroni post hoc analyses, as appropriate. A $\mathrm{P}<0.05$ was considered to indicate a statistically significant difference. All statistical analyses were performed using the SPSS 13.0 software (SPSS, Inc., Chicago, IL, USA).

\section{Results}

Effect of miR-7-5p on proliferation and migration of LS174T cells. The proliferation and migration functions of intestinal epithelial cells are significant following intestinal damage. The effect of miR-7-5p in the proliferation of LS174T cells was evaluated using the MTT assay, and the effect of miR-7-5p on the migration of LS174T cells was evaluated in Transwell chambers. Fig. 1 demonstrates that successful results were obtained $24 \mathrm{~h}$ after transfecting miR-7-5p mimics and a miR-7-5p inhibitor. As presented in Fig. 2, compared with the NC group, the miRNA mimic NC group and the miRNA 

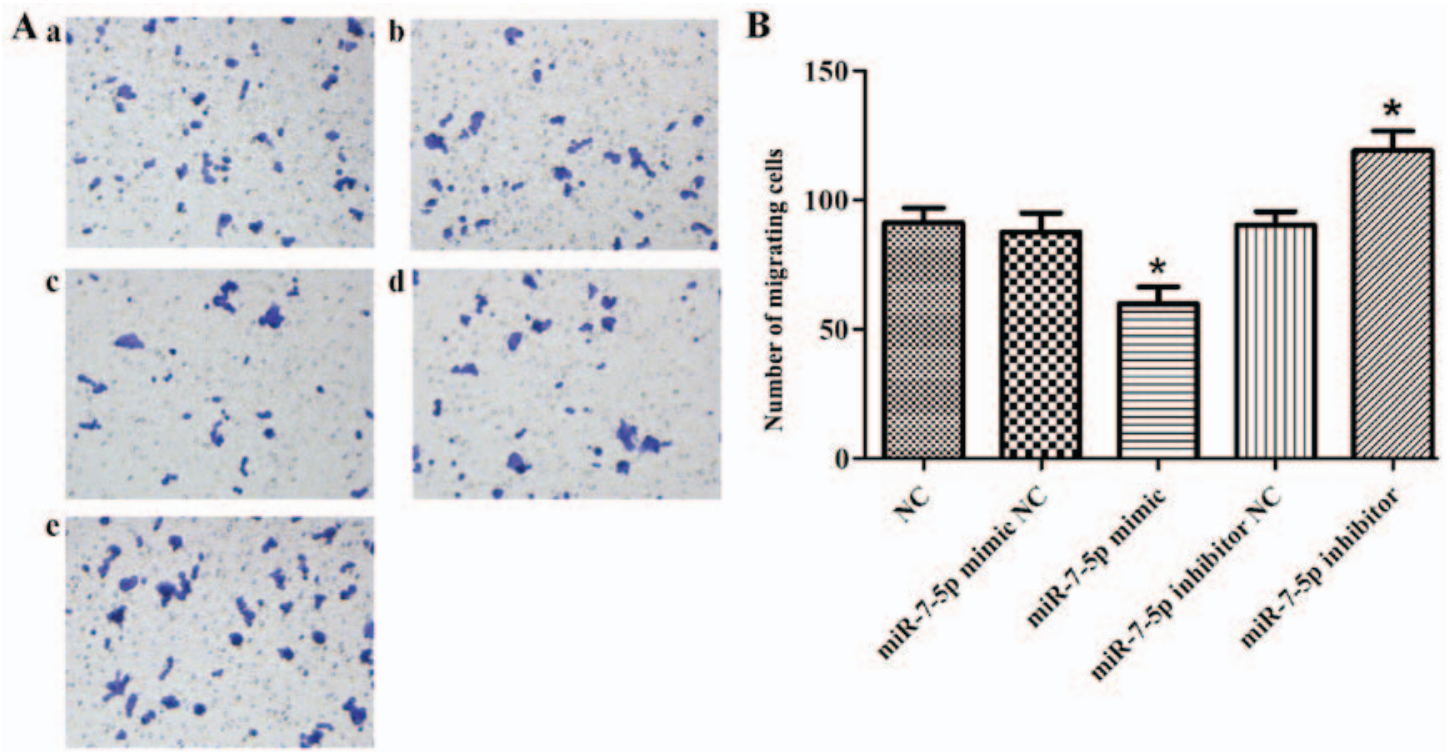

Figure 3. Effects of miR-7-5p on migration of LS174T cells. The Transwell assay was performed to determine the effect of overexpression and downregulation of miR-7-5p on migration of LS174T cells. (A) Crystal violet staining results of LS174T cell migration through the Transwell chamber (magnification, $x 200$ ): (a) LS174T cells transfected with NC; (b) LS174T cells transfected with miR-7-5p mimic NC; (c) LS174T cells transfected with miR-7-5p mimics; (d) LS174T cells transfected with miR-7-5p inhibitor NC; (e) LS174T cells transfected with miR-7-5p inhibitor. The assays were performed in triplicate. (B) Histogram of the number of LS174T cells migrating through the Transwell chamber. The assays were performed in triplicate. All values are presented as the means \pm standard deviation of three replicates. " $\mathrm{P}<0.05$ compared with NC, miR-7-5p mimic NC and miR-7-5p inhibitor NC groups. miR, microRNA; NC, negative control.

$\mathbf{A}$

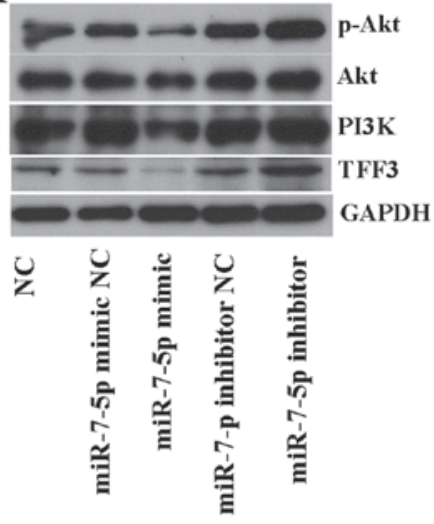

B

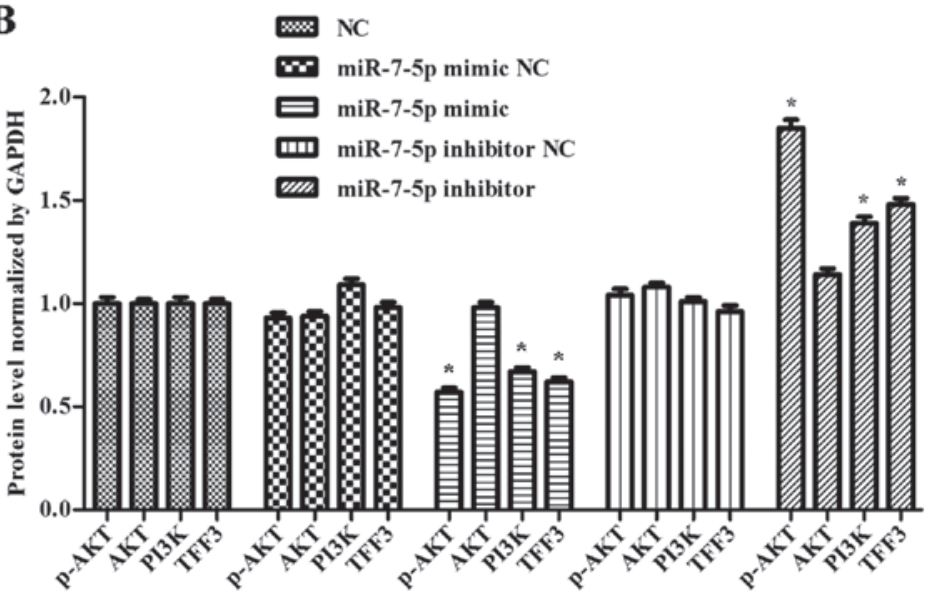

Figure 4. (A) Western blot detection of TFF3, PI3K, Akt and p-Akt expression levels in cells with overexpressed or downregulated miR-7-5p levels. GAPDH served as the loading control. (B) Quantitative analysis of TFF3, PI3K, Akt and p-Akt protein levels normalized to GAPDH levels. The assays were performed in triplicate. All values are presented as the means \pm standard deviation of three replicates. ${ }^{*} \mathrm{P}<0.05$ compared with NC, miR-7-5p mimic NC and miR-7-5p inhibitor NC groups. TFF3, trefoil factor 3; PI3K, phosphoinositide 3-kinase; p, phosphorylated; miR, microRNA; GAPDH, glyceraldehydes-3-phosphate dehydrogenase.

inhibitor NC group, the overexpression of miR-7-5p decreased the cell proliferation ability $(\mathrm{P}<0.05)$, whereas inhibiting the expression of miR-7-5p increased cell proliferation. Fig. 3 demonstrates that, compared with the NC group, the miRNA mimic NC group and the miRNA inhibitor NC group, the overexpression of miR-7-5p decreased, whereas inhibiting the expression level of miR-7-5p increased the number of cells that migrated through the chamber $(\mathrm{P}<0.05)$.

Effect of miR-7-5p on the expression levels of TFF3, PI3K, Akt and p-Akt protein. As shown in Fig. 4, compared with the NC group, the miRNA mimic NC group and the miRNA inhibitor $\mathrm{NC}$ group, the overexpression of miR-7-5p decreased the levels of TFF3, PI3K and p-Akt protein expression, whereas inhibiting the expression level of miR-7-5p increased TFF3, PI3K and p-Akt protein expression levels and the difference was statistically significant $(\mathrm{P}<0.05)$. However, no effect of miR-7-5p was observed on the expression level of the Akt protein. In a previous study, miR-7-5p was demonstrated to bind to the 3'UTR of TFF3. Thus, these results indicated that miR-7-5p influences the proliferation and migration of LS174T cells by targeting TFF3, with the possible participation of the PI3K/Akt signalling pathway.

Effect of miR-7-5p, TFF3 and LY294002 on the protein expression levels of TFF3, PI3K, Akt and p-Akt. According to 


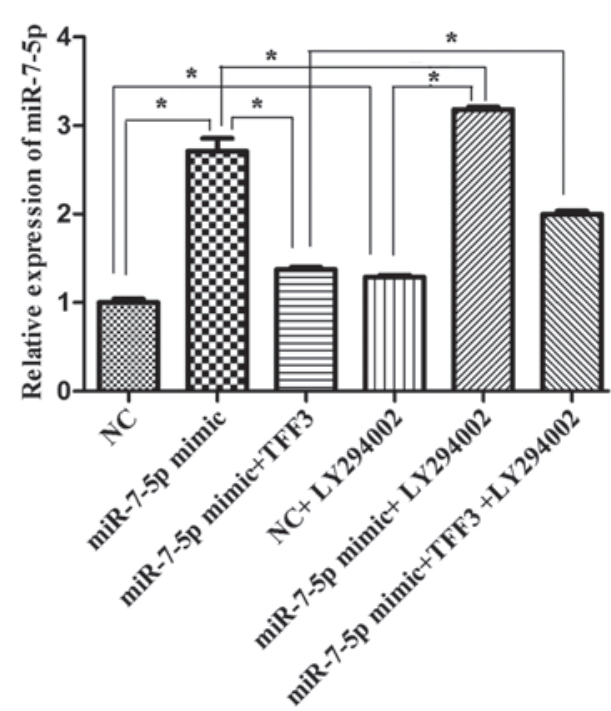

Figure 5. Expression levels of miR-7-5p following transfection with miR-7-5p mimics, TFF3 plasmid and LY294002 were detected by reverse transcription-quantitative polymerase chain reaction. U6 served as a control. The assays were performed in triplicate. All values are presented as the means \pm standard deviation of three replicates. ${ }^{*} \mathrm{P}<0.05$. miR, microRNA; $\mathrm{TFF} 3$, trefoil factor 3 ; $\mathrm{NC}$, negative control.

the experimental groups, the transfected cells were harvested $48 \mathrm{~h}$ after transfection, and the mRNA expression level of miR-7 was detected via qPCR. As demonstrated in Fig. 5, the expression level of miR-7-5p in the miR-7-5p mimic group was significantly higher than that in the miRNA NC group. Compared with the miR-7-5p mimic group, the expression level of miR-7-5p in the miR-7-5p mimic + TFF3 plasmid group was significantly decreased, while compared with the miR-7-5p mimic + TFF3 plasmid group, the expression level of miR-7-5p in the miR-7-5p mimic + TFF3 plasmid + LY294002 group was significantly increased, and this difference was statistically significant $(\mathrm{P}<0.05)$. These findings indicated that the TFF3 overexpression plasmid was able to inhibit the expression level of miR-7-5p, but that LY294002 inhibited this inhibitory effect of the TFF3 plasmid on miR-7-5p. The expression level of miR-7-5p in the miRNA NC + LY294002 group, the miR-7-5p mimic + LY294002 group, and the miR-7-5p mimic + TFF3 plasmid + LY294002 group was greater than that in the miRNA NC group, the miR-7-5p mimic group, and the miR-7-5p mimic + TFF3 plasmid group, respectively, and these differences were statistically significant $(\mathrm{P}<0.05)$. Thus, these findings indicated that the expression level of miR-7-5p was upregulated by the PI3K/Akt signalling pathway inhibitor, indicating that inhibition of the PI3K/Akt signalling pathway may result in feedback upregulation of miR-7-5p expression.

The TFF3 protein was detected by western blot analysis (Fig. 6). The results (Figs. 6-8) showed that the expression levels of the TFF3, PI3K, and p-Akt proteins in the miR-7-5p mimic group were significantly lower than those in the NC group, whereas the expression levels of the TFF3, PI3K and p-Akt proteins in the miR-7-5p mimic + TFF3 plasmid group were higher than those in the miR-7-5p mimic group. The difference between these groups was significant $(\mathrm{P}<0.05)$, indicating that miR-7-5p downregulates the protein expression of its target, TFF3. The TFF3 promotes PI3K protein expression,
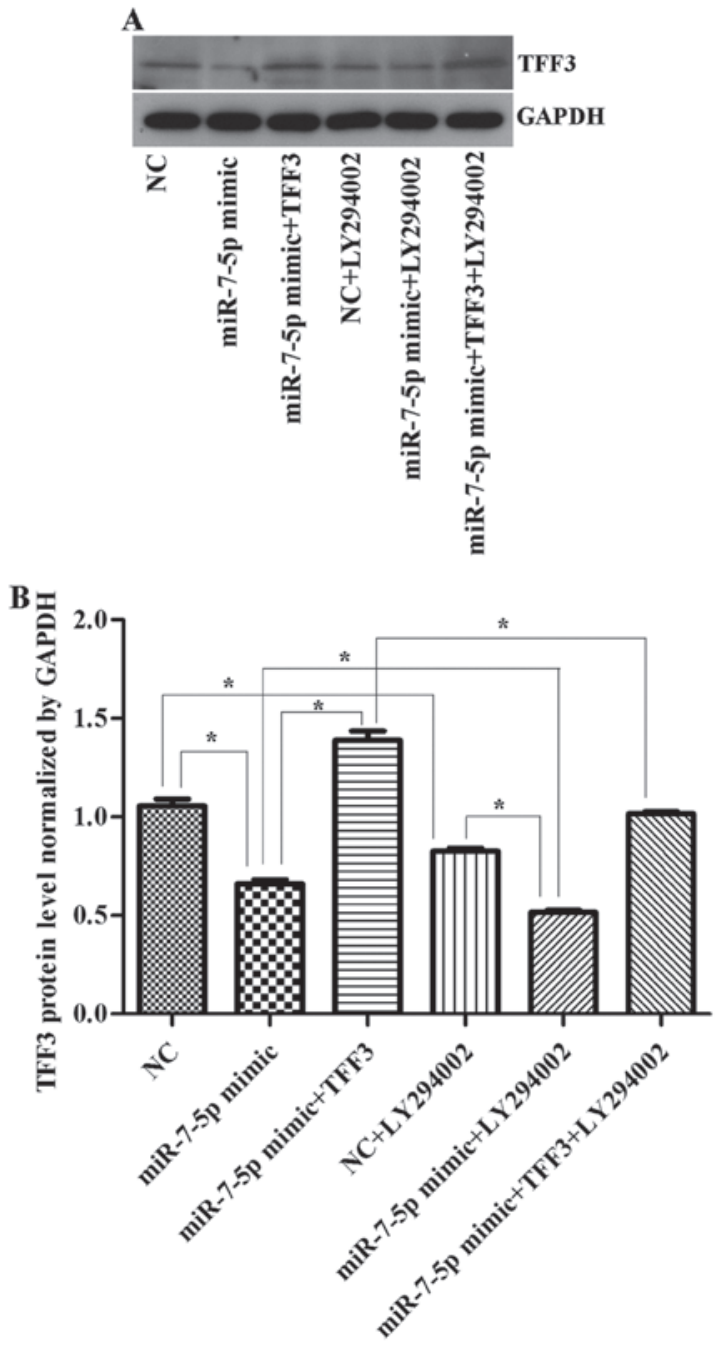

Figure 6. (A) Western blot detection of TFF3 expression levels in cells following transfection with miR-7-5p mimics, TFF3 plasmid and LY294002. GAPDH served as the loading control. (B) Quantitative analysis of TFF3 protein expression levels normalized to GAPDH levels. The assays were performed in triplicate. All values are presented as the means \pm standard deviation of three replicates. ${ }^{*} \mathrm{P}<0.05$. TFF3, trefoil factor 3 ; miR, microRNA; GAPDH, glyceraldehydes-3-phosphate dehydrogenase; NC, negative control.

and miR-7-5p inhibits the activation of the PI3K/Akt signalling pathway while downregulating TFF3 protein expression levels. However, following treatment of the miR-7-5p mimic + TFF3 plasmid group with LY294002, the expression levels of the TFF3, PI3K and p-Akt proteins decreased, indicating that blocking the PI3K/Akt signalling pathway further promotes the effect of miR-7-5p on TFF3. Compared with the NC group, the expression level of the TFF3 protein in the $\mathrm{NC}+$ LY294002 group was significantly decreased $(\mathrm{P}<0.05)$. Compared with the miR-7-5p mimic group, the expression level of the TFF3 protein in miR-7-5p mimics + LY294002 group was significantly decreased $(\mathrm{P}<0.05)$. Compared with the miR-7-5p mimic + TFF3 plasmid group, the expression level of the TFF3 protein in the miR-7-5p mimic + TFF3 plasmid + LY294002 group was also significantly decreased $(\mathrm{P}<0.05)$. Taken together, these findings demonstrated that subsequent to adding the PI3K/Akt signalling pathway inhibitor, the expression level of miR-7-5p was upregulated and the expression level of TFF3 was downregulated, which 
A

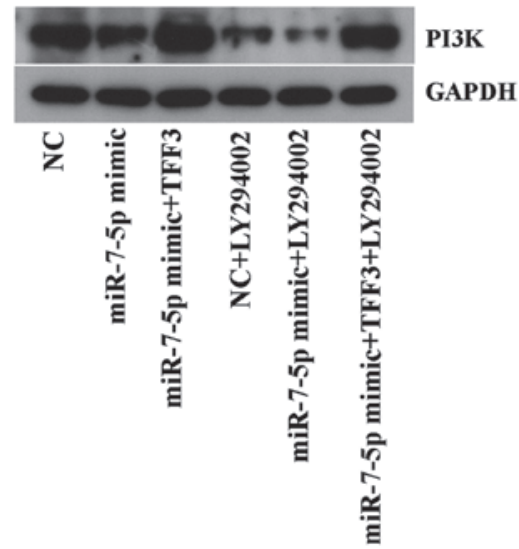

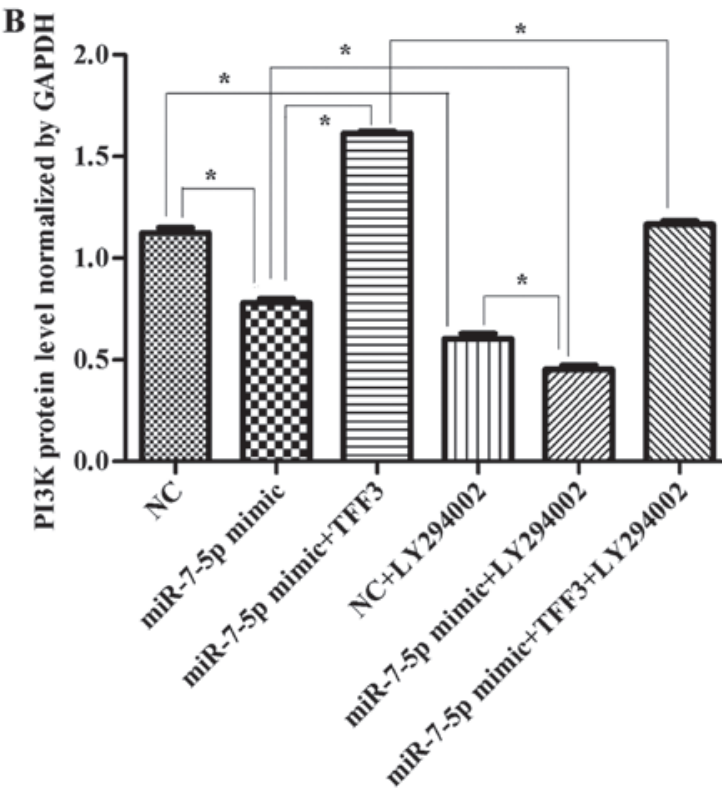

Figure 7. (A) Western blot detection of PI3K expression levels in cells following transfection with miR-7-5p mimics, TFF3 plasmid and LY294002. GAPDH served as the loading control. (B) Quantitative analysis of PI3K protein expression levels normalized to GAPDH levels. The assays were performed in triplicate. All values are presented as the means \pm standard deviation of three replicates. "P $<0.05$. PI3K, phosphoinositide 3-kinase; TFF3, trefoil factor 3; miR, microRNA; GAPDH, glyceraldehydes-3-phosphate dehydrogenase; NC, negative control.
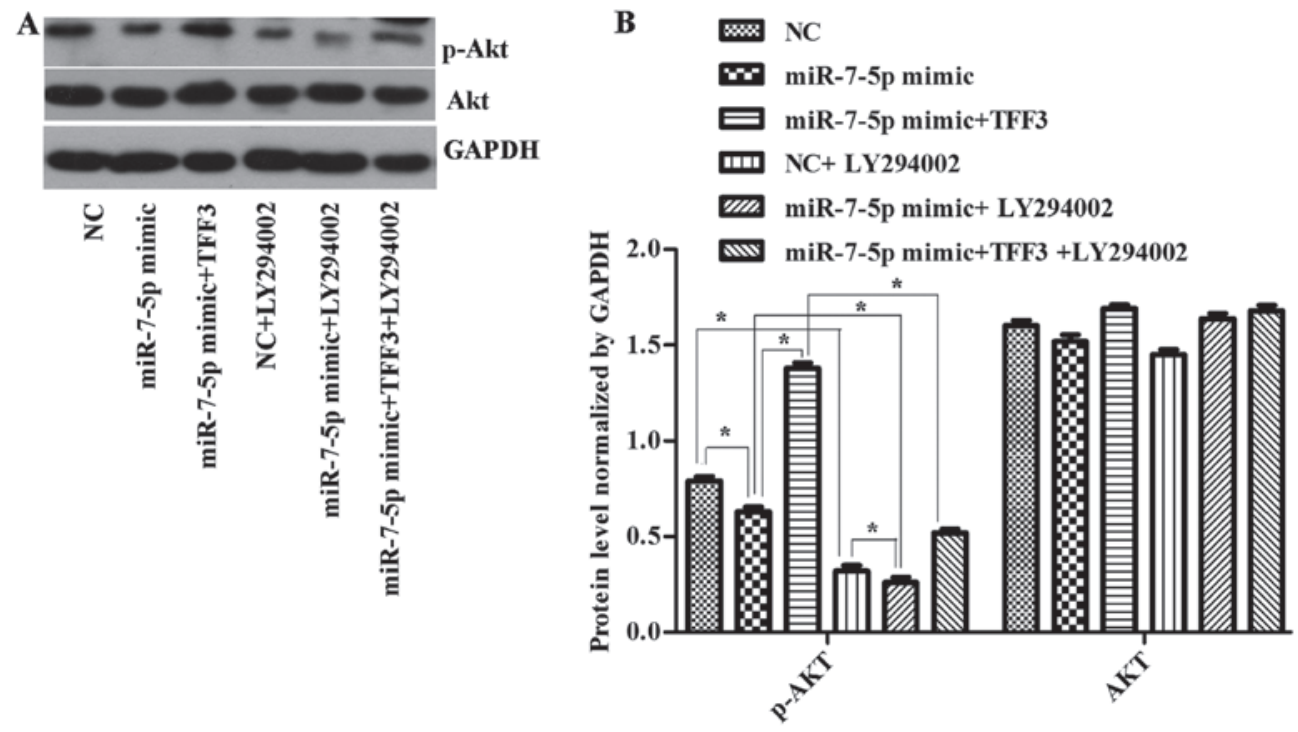

Figure 8. (A) Western blot detection of Akt and p-Akt levels in cells following transfection with miR-7-5p mimics, TFF3 plasmid and LY294002. GAPDH served as the loading control. (B) Quantitative analysis of Akt and p-Akt protein expression levels normalized to GAPDH levels. The assays were performed in triplicate. All values are presented as the means \pm standard deviation of three replicates. ${ }^{*} \mathrm{P}<0.05$. p, phosphorylated; TFF3, trefoil factor 3; miR, microRNA; GAPDH, glyceraldehydes-3-phosphate dehydrogenase; NC, negative control.

indicated that inhibiting the PI3K/Akt signalling pathway may exert a feedback effect leading to the upregulation of miR-7-5p expression and the inhibition of TFF3 expression.

The expression levels of the PI3K and p-Akt protein were consistent with the trend of TFF3 protein expression (Figs. 7 and 8), although there was no significant difference in the expression levels of the AKT protein between the groups (Fig. 8B), suggesting that the primary mechanism by which miR-7-5p regulates the expression level of TFF3 and affects the PI3K/Akt signalling pathway occurs via modulation of Akt protein phosphorylation.
Effect of miR-7-5p, TFF3 and LY294002 on the proliferation and migration of LS174T cells. To detect the cell proliferation of each group, the cell optical density (OD) at $490 \mathrm{~nm}$ was detected at $0,24,48$ and $72 \mathrm{~h}$ after transfection. As shown in Fig. 9, compared with the NC group, the OD value of the miR-7 mimic group decreased significantly, indicating a decreased cell proliferation ability; the difference between the groups was statistically significant $(\mathrm{P}<0.05)$. Compared with the miR-7-5p mimic group, the miR-7-5p mimic + TFF3 plasmid group showed a significantly increased OD value and enhanced cell proliferation ability, with a significant difference 


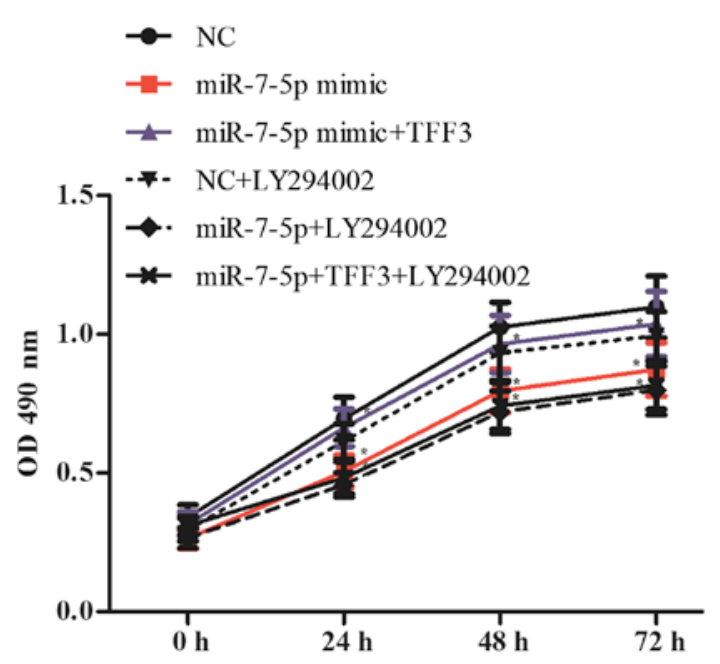

Figure 9. Effects of miR-7-5p, TFF3 and LY294002 on the proliferation of LS174T cells. The assays were performed in triplicate. All values are presented as the means \pm standard deviation of three replicates. ${ }^{*} \mathrm{P}<0.05$. miR, microRNA; TFF3, trefoil factor 3; OD, optical density; NC, negative control.

observed between the groups $(\mathrm{P}<0.05)$. Compared with the miR-7-5p mimic group, the miR-7-5p mimic + LY294002 group exhibited a slight decrease in the OD value, although the difference between the groups was not significant $(\mathrm{P}>0.05)$. This result indicates that in addition to the PI3K/Akt signalling pathway, miR-7-5p affects cell proliferation via pathways other than regulating TFF3. However, compared with the miR-7-5p mimic + TFF3 plasmid group, the miR-7-5p mimic + TFF3 plasmid + LY294002 group exhibited a decreased OD value and decreased cell proliferation ability. These results indicated that the PI3K/Akt signalling pathway is involved in the regulation of TFF3 by miR-7-5p, subsequently affecting the proliferation of LS174T cells, and that blocking the PI3K/Akt signalling pathway further promotes the inhibitory effect of miR-7-5p on cell proliferation.

The present study illustrated the effect of miR-7-5p, TFF3 and LY294002 on the migration of LS174T cells via Transwell chamber assays. The number of cells that migrated through the Transwell chamber was counted following $24 \mathrm{~h}$ of incubation. As presented in Fig. 10, compared with the NC group, the cell migration ability of the miR-7-5p high expression group was significantly decreased. Compared with the miR-7-5p mimic group, the number of cells passing through the Transwell membrane was increased in the miR-7-5p mimic + TFF3 plasmid group. This finding indicated that the cell migration ability was increased, indicating that the TFF3 plasmid weakened the inhibitory effect of miR-7-5p on the migration ability of the cells, and supported the effect of miR-7-5p by targeting TFF3 and mediating cell migration. Compared with the miR-7-5p mimic group, the miR-7-5p mimic + LY294002 group exhibited a decrease in the number of cells passing through the Transwell membrane, suggesting that LY294002 further downregulated the migration ability of LS174T cells. The number of cells passing through the Transwell membrane in the miR-7-5p mimic group decreased, whereas it increased in the miR-7-5p mimic + TFF3 plasmid group. However, when the miR-7-5p mimic + TFF3 overexpression plasmid group was treated with LY294002, the number of
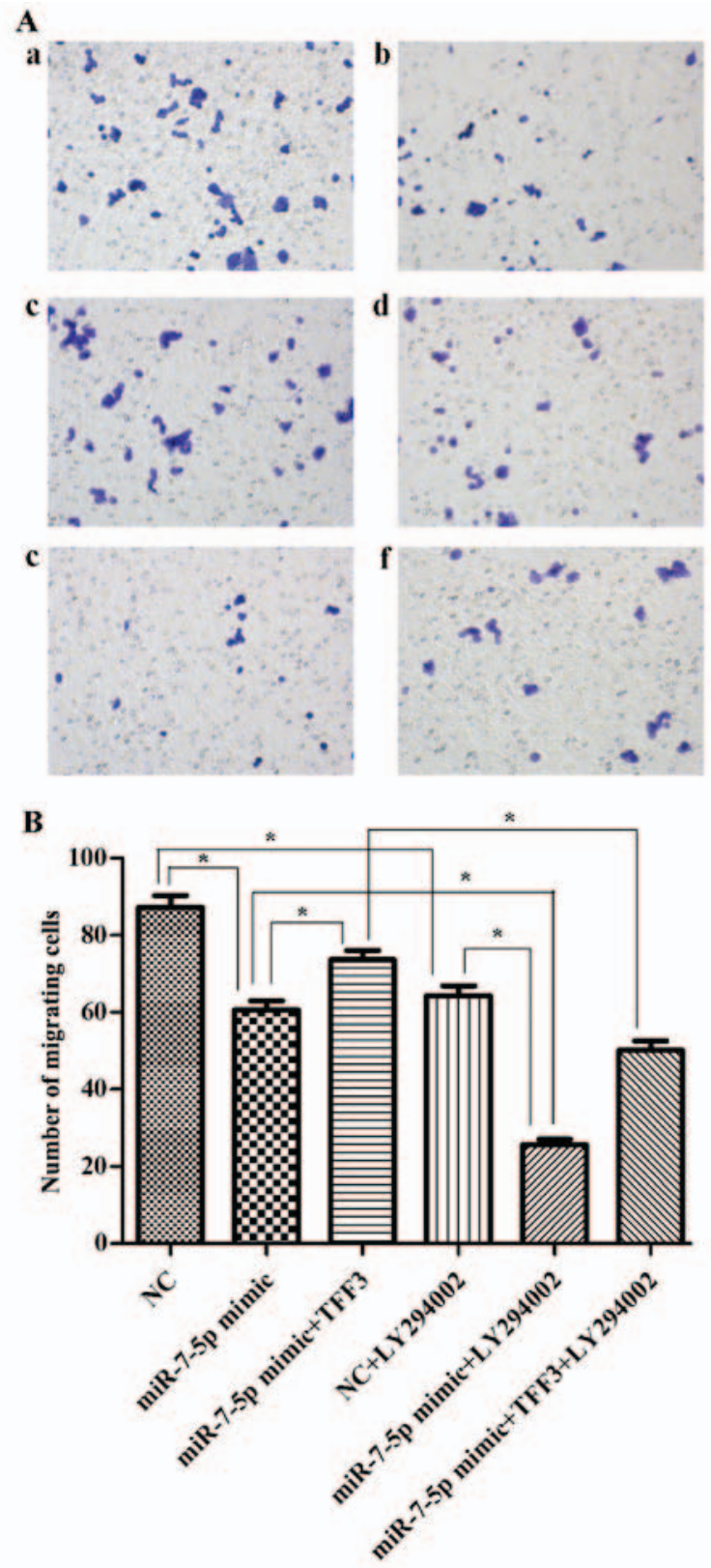

Figure 10. Effects of miR-7-5p, TFF3 and LY294002 on the migration of LS174T cells. (A) The crystal violet staining results of LS174T cell migration through the Transwell chamber (magnification, x200): (a) LS174T cells transfected with miR-7-5p mimic NC; (b) LS174T cells transfected with miR-7-5p mimics; (c) LS174T cells transfected with miR-7-5p mimics and TFF3 plasmid; (d) LS174T cells transfected with miR-7-5p mimic NC and LY294002; (e) LS174T cells transfected with miR-7-5p mimics and LY294002; (f) LS174T cells transfected with miR-7-5p mimics, TFF3 plasmid and LY294002. (B) Histogram of the number of LS174T cells migrating through the Transwell chamber. The assays were performed in triplicate. All values are presented as the means \pm standard deviation of three replicates. miR. ${ }^{*} \mathrm{P}<0.05$. miR, microRNA; TFF3, trefoil factor 3; NC, negative control.

cells passing through the Transwell membrane decreased once more, indicating that LY294002 blocked the promoting effect of the TFF3 overexpression plasmid on cell migration. Taken together, these findings indicate that the effect of miR-7-5p on the migration of LS174T cells via TFF3 regulation was mediated by the PI3K/Akt signalling pathway, and blocking this pathway may further promote the inhibitory effect of miR-7-5p on cell migration. 


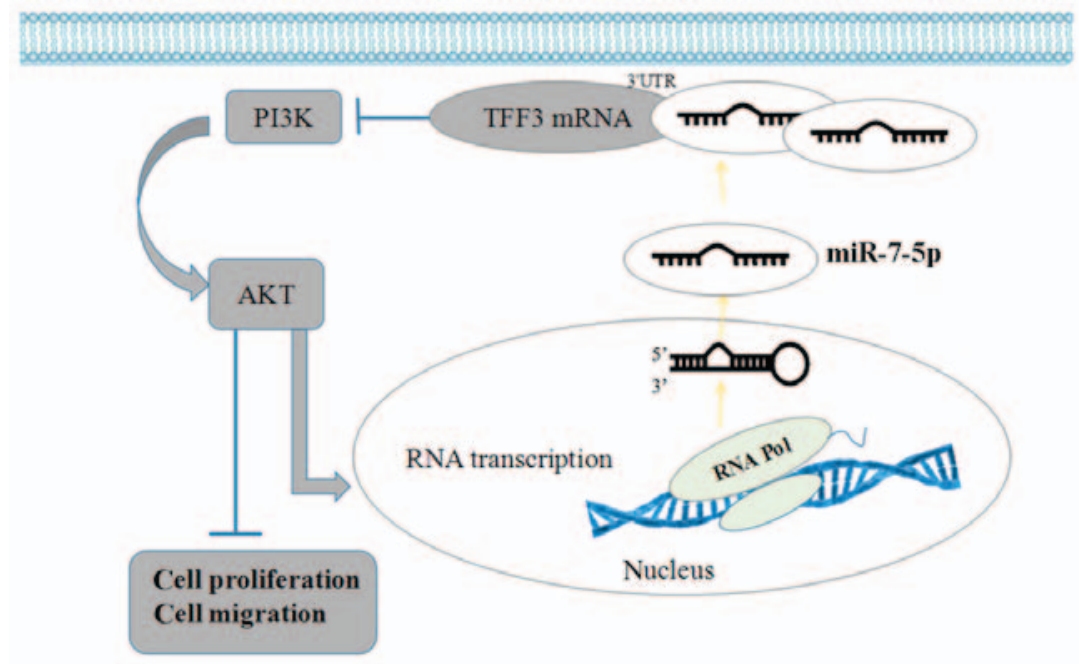

Figure 11. Interaction diagram of miR-7-5p, TFF3 and the PI3K/Akt signalling pathway when miR-7-5p regulates the proliferation and migration of LS174T cells. miR, microRNA; TFF3, trefoil factor 3; PI3K, phosphoinositide 3-kinase; 3'UTR, 3'-untranslated region.

\section{Discussion}

miRNAs are a type of snRNA that lead to mRNA degradation and translational repression by binding to the 3'UTR of target mRNAs. miRNAs are significant in processes, such as cell proliferation, apoptosis, growth and development, cell differentiation and metabolism (17).

miR-7 is one type of mature miRNA that has been investigated in tumour diseases. Numerous reports have suggested that miR-7 affects the functions of cell metabolism, growth, proliferation and apoptosis by acting on different target proteins $(14,15,18-20)$. Fang et al (18) proposed that miR-7 inhibits the growth and metabolism of hepatocellular carcinoma by regulating the PI3K/Akt signalling pathway. Meza-Sosa et al (19) demonstrated that miR-7 promotes epithelial cell transdifferentiation by targeting Kruppel like factor 4. Xu et al (20) revealed that miR-7 regulates XRCC2, and thereby inhibits the proliferation of colorectal cancer cells and induces apoptosis (20). In our previous study, miR-7-5p was found to be differentially expressed in IBD lesions and normal tissues. In addition, it was confirmed that miR-7-5p binds to the TFF3 3'UTR and regulates the expression level of TFF3 at the post-transcriptional level (13).

The TFF3 proteins are a group of small molecular peptides that are predominantly secreted by goblet cells (21). TFF3 exhibits a special three-lobed structure. This structure exhibits high stability, which causes the TFF to present strong anti-protease hydrolysis, acid digestibility and heat-resistant properties. Therefore, TFF3 maintains biological activity in the complex environment of the digestive tract (22). Investigation of the response of TFF3 to mucosal damage and its response in patients with IBD has indicated that TFF3 is significant in injury repair and mucosal protection, and the underlying mechanisms include the promotion of cell migration, cell proliferation and anti-apoptosis effects (23-25).

The present study demonstrates that miR-7 may regulate the function of tumour cells by regulating multiple pathways, such as the PI3K/Akt and mitogen-activated protein kinase kinase/ERK signalling pathways $(26,27)$. Li et al $(28)$ found that miR-7 inhibits the proliferation and invasion of human colorectal cancer cells, and that PI3K signalling pathways are involved in this process. Zhang et al (29) showed that miR-21 could regulate the tight junctional permeability of intestinal epithelial cells, which was mediated by the PI3K/Akt signalling pathway. It has also been suggested that TFF3 and the PI3K/Akt pathway play important roles in cell migration and proliferation. Dise et al (30) demonstrated that EGF promoted intestinal epithelial cell migration by activating PI3K and causing Rac activation. Langlois et al (31) showed that PTEN inhibits tumour progression by controlling cell polarity, the establishment of cell-cell junctions, paracellular permeability, migration and metabolic potential and that PTEN phosphorylation is mediated by the activation of PI3K. Sun et al (32) demonstrated that intestinal trefoil factor (ITF) can promote the proliferation and migration of gastric epithelial cells. ITF protects the integrity of gastric epithelial cells from damage by activating PI3K/Akt cell signalling pathways. Lin et al (33) revealed that TFF3 overexpression may promote the expression of zonula occludens-1, occludin and claudin-1, but this effect was inhibited after suppressing the PI3K/Akt signalling pathway.

Therefore, the aim of the present study was to elucidate the association between miR-7-5p, TFF3 and PI3K/Akt in the process, whereby miR-7-5p regulates the proliferation and migration of intestinal epithelial cell. The current results revealed that miR-7-5p decreased the expression level of TFF3, and inhibited LS174T cell proliferation and migration, accompanied by decreased expression of PI3K and p-Akt. Furthermore, it was identified that miR-7-5p expression levels were decreased following combined treatment with the TFF3 plasmid and miR-7-5p mimics, compared with treatment with the miR-7-5p mimics alone. This effect was accompanied by increased expression levels of TFF3, PI3K and p-Akt, and by enhanced LS174T cell proliferation and migration. Furthermore, the expression level of miR-7-5p in the miRNA $\mathrm{NC}+$ LY294002 group, the miR-7-5p mimic + LY294002 group, and the miR-7-5p mimic + TFF3 plasmid + LY294002 group was higher than in the NC group, the miR-7-5p mimic 
group, and the miR-7-5p mimic + TFF3 plasmid group, respectively. Accordingly, the expression level of TFF3 was downregulated, and cell proliferation and migration were downregulated simultaneously. These data indicate that miR-7-5p regulates TFF3 and inhibits the proliferation and migration of LS174T cells. The PI3K/Akt signalling pathway is involved in the regulation of cell proliferation and migration via miR-7-5p targeting TFF3 (Fig. 11). The PI3K/Akt signalling pathway may exert a feedback regulation effect on miR-7-5p; inhibition of the activity of this pathway may enhance miR-7-5p expression levels and further enhance the effect of miR-7-5p on cell proliferation and migration.

In conclusion, miR-7-5p inhibits the proliferation and migration of LS174T cells by targeting TFF3 via inhibiting the PI3K/Akt signalling pathway. Additionally, the PI3K/Akt signalling pathway may have a feedback regulation effect on miR-7-5p. Therefore, miR-7-5p may serve as a therapeutic target for protection of intestinal epithelial barrier integrity.

\section{Acknowledgements}

The present study was supported by grants from the National Natural Science Foundation of China Youth Foundation (grant no. 81400585) and the Natural Science Foundation of Liaoning Province, China (grant no. 2014021042).

\section{References}

1. Peloquin JM, Goel G, Villablanca EJ and Xavier RJ: Mechanisms of pediatric inflammatory bowel disease. Annu Rev Immunol 34 31-64, 2016.

2. Neurath MF and Travis SP: Mucosal healing in inflammatory bowel diseases: A systematic review. Gut 61: 1619-1635, 2012.

3. Hollander D, Vadheim CM, Brettholz E, Petersen GM, Delahunty $\mathrm{T}$ and Rotter JI: Increased intestinal permeability in patients with Crohn's disease and their relatives. A possible etiologic factor. Ann Intern Med 105: 883-885, 1986.

4. Petersson J, Schreiber O, Hansson GC, Gendler SJ, Velcich A, Lundberg JO, Roos S, Holm L and Phillipson M: Importance and regulation of the colonic mucus barrier in a mouse model of colitis. Am J Physiol Gastrointest Liver Physiol 300: G327-G333, 2011.

5. Su L, Shen L, Clayburgh DR, Nalle SC, Sullivan EA, Meddings JB, Abraham C and Turner JR: Targeted epithelia tight junction dysfunction causes immune activation and contributes to development of experimental colitis. Gastroenterology 136: 551-563, 2009.

6. Rhoads JM, Niu X, Odle J and Graves LM: Role of mTOR signaling in intestinal cell migration. Am J Physiol Gastrointest Liver Physiol 291: G510-G517, 2006.

7. Sukhotnik I, Shteinberg D, Ben Lulu S, Bashenko Y, Mogilner JG, Ure BM, Shaoul R, Shamian B and Coran AG: Transforming growth factor-alpha stimulates enterocyte proliferation and accelerates intestinal recovery following methotrexate-induced intestinal mucositis in a rat and a cell culture model. Pediatr Surg Int 24: 1303-1311, 2008.

8. Hoffmann W: Trefoil factors TFF (trefoil factor family) peptidetriggered signals promoting mucosal restitution. Cell Mol Life Sci 62: 2932-2938, 2005.

9. Kjellev S: The trefoil factor family - small peptides with multiple functionalities. Cell Mol Life Sci 66: 1350-1369, 2009.

10. Sturm A and Dignass AU: Epithelial restitution and wound healing in inflammatory bowel disease. World J Gastroenterol 14: 348-353, 2008.

11. Perrais $M$, Chen $X$, Perez-Moreno $M$ and Gumbiner BM: E-cadherin homophilic ligation inhibits cell growth and epidermal growth factor receptor signaling independently of other cell interactions. Mol Biol Cell 18: 2013-2025, 2007.

12. Oloumi A, McPhee T and Dedhar S: Regulation of E-cadherin expression and beta-catenin/Tcf transcriptional activity by the integrin-linked kinase. Biochim Biophys Acta 1691: 1-15, 2004.
13. Guo J, Sun M, Teng X and Xu L: MicroRNA-7-5p regulates the expression of TFF3 in inflammatory bowel disease. Mol Med Rep 16: 1200-1206, 2017.

14. Giles KM, Brown RA, Ganda C, Podgorny MJ, Candy PA, Wintle LC, Richardson KL, Kalinowski FC, Stuart LM, Epis MR, et al: microRNA-7-5p inhibits melanoma cell proliferation and metastasis by suppressing RelA/NF- $\kappa$ B. Oncotarget 7: 31663-31680, 2016.

15. Kefas B, Godlewski J, Comeau L, Li Y, Abounader R, Hawkinson M, Lee J, Fine H, Chiocca EA, Lawler S, et al: microRNA-7 inhibits the epidermal growth factor receptor and the Akt pathway and is down-regulated in glioblastoma. Cancer Res 68: 3566-3572, 2008

16. Livak KJ and Schmittgen TD: Analysis of relative gene expression data using real-time quantitative PCR and the 2(-Delta Delta C(T)) Method. Methods 25: 402-408, 2001.

17. Ambros V: The functions of animal microRNAs. Nature 431: 350-355, 2004.

18. Fang Y, Xue JL, Shen Q, Chen J and Tian L: MicroRNA-7 inhibits tumor growth and metastasis by targeting the phosphoinositide 3-kinase/Akt pathway in hepatocellular carcinoma. Hepatology 55: 1852-1862, 2012

19. Meza-Sosa KF, Pérez-García EI, Camacho-Concha N, LópezGutiérrez O, Pedraza-Alva G and Pérez-Martínez L: MiR-7 promotes epithelial cell transformation by targeting the tumor suppressor KLF4. PLoS One 9: e103987, 2014.

20. Xu K, Chen Z, Qin C and Song X: miR-7 inhibits colorectal cancer cell proliferation and induces apoptosis by targeting XRCC2. Onco Targets Ther 7: 325-332, 2014.

21. Meyer zum Büschenfelde D, Hoschützky H, Tauber R and Huber O: Molecular mechanisms involved in TFF3 peptidemediated modulation of the E-cadherin/catenin cell adhesion complex. Peptides 25: 873-883, 2004.

22. Buda A, Jepson MA and Pignatelli M: Regulatory function of trefoil peptides (TFF) on intestinal cell junctional complexes. Cell Commun Adhes 19: 63-68, 2012.

23. Hoffmann W: Trefoil factor family (TFF) peptides: Regulators of mucosal regeneration and repair, and more. Peptides 25: 727-730, 2004.

24. Wright NA, Poulsom R, Stamp G, Van Norden S, Sarraf C, Elia G, Ahnen D, Jeffery R, Longcroft J, Pike C, et al: Trefoil peptide gene expression in gastrointestinal epithelial cells in inflammatory bowel disease. Scand J Gastroenterol Suppl 193 (sup193): 76-82, 1992.

25. Renes IB, Verburg M, Van Nispen DJ, Büller HA, Dekker J and Einerhand AW: Distinct epithelial responses in experimental colitis: Implications for ion uptake and mucosal protection. Am J Physiol Gastrointest Liver Physiol 283: G169-G179, 2002.

26. Zhou X, Hu Y, Dai L, Wang Y, Zhou J, Wang W, Di W and Qiu L: MicroRNA-7 inhibits tumor metastasis and reverses epithelialmesenchymal transition through AKT/ERK1/2 inactivation by targeting EGFR in epithelial ovarian cancer. PLoS One 9: e96718, 2014.

27. Liu Z, Jiang Z, Huang J, Huang S, Li Y, Yu S, Yu S and Liu X: miR-7 inhibits glioblastoma growth by simultaneously interfering with the PI3K/ATK and Raf/MEK/ERK pathways. Int J Oncol 44: 1571-1580, 2014.

28. Li Y, Li Y, Liu Y, Xie P, Li F and Li G: PAX6, a novel target of microRNA-7, promotes cellular proliferation and invasion in human colorectal cancer cells. Dig Dis Sci 59: 598-606, 2014.

29. Zhang L, Shen J, Cheng J and Fan X: MicroRNA-21 regulates intestinal epithelial tight junction permeability. Cell Biochem Funct 33: 235-240, 2015.

30. Dise RS, Frey MR, Whitehead RH and Polk DB: Epidermal growth factor stimulates Rac activation through Src and phosphatidylinositol 3-kinase to promote colonic epithelial cell migration. Am J Physiol Gastrointest Liver Physiol 294: G276-G285, 2008

31. Langlois MJ, Bergeron S, Bernatchez G, Boudreau F, Saucier C, Perreault N, Carrier JC and Rivard N: The PTEN phosphatase controls intestinal epithelial cell polarity and barrier function: Role in colorectal cancer progression. PLoS One 5: e15742, 2010

32. Sun Z, Liu H, Yang Z, Shao D, Zhang W, Ren Y, Sun B, Lin J, $\mathrm{Xu} \mathrm{M}$ and Nie S: Intestinal trefoil factor activates the PI3K/Akt signaling pathway to protect gastric mucosal epithelium from damage. Int J Oncol 45: 1123-1132, 2014.

33. Lin N, Xu LF and Sun M: The protective effect of trefoil factor 3 on the intestinal tight junction barrier is mediated by toll-like receptor 2 via a PI3K/Akt dependent mechanism. Biochem Biophys Res Commun 440: 143-149, 2013. 